\title{
APLICACIÓN DEL MODELO UNDERSTANDING BY DESIGN EN ESTADÍSTICA EN LA PREPARATORIA ENRIQUE CABRERA BARROSO DE LA BENEMÉRITA UNIVERSIDAD AUTÓNOMA DE PUEBLA
}

\author{
Martha García C., Guillermina Sánchez L. \\ Benemérita Universidad Autónoma de Puebla \\ marthamath@yahoo.com.mx
}

El presente trabajo es un estudio cualitativo aplicado a 72 estudiantes de Nivel Medio Superior, que describe los resultados de la aplicación del modelo Understanding by Design (UbD) a través de actividades generadoras como lo es, la elaboración de un cuestionario, con el propósito de propiciar en el alumno la potenciación de las habilidades básicas de pensamiento; para que, a partir de la observación pueda comparar, analizar y sintetizar información estadística necesaria y así, deducir las preguntas óptimas que deben realizarse para generar las gráficas mostradas. Concluyendo que cuando el estudiante observa pertinentemente las gráficas de un estudio estadístico y es capaz de deducir las preguntas de la encuesta de aplicación, entonces crea conclusiones que revelan una adecuada lectura e interpretación de gráficas.

\section{ANTECEDENTES}

En la Preparatoria Enrique Cabrera Barroso Urbana de la Benemérita Universidad Autónoma de Puebla (BUAP) la planificación es anual, y en el tercer año los grupos se integran de acuerdo al interés vocacional de cada estudiante por lo que quedan divididos en 4 áreas: Salud, Ingenierías, Humanidades y Ciencias Sociales.

La materia de Estadística se imparte sólo a las áreas de Humanidades y Ciencias Sociales, por lo que el trabajo gira en torno a los grupos de 3er. Año pertenecientes a estas áreas. Los programas de Estudio del Nivel Medio Superior de la BUAP se encuentran adaptados a Competencias en su reforma bajo el Plan "Minerva", específicamente para el bachillerato son denominados como Plan 06 por Competencias. En los bloques V y VI del programa de Estadística Descriptiva, de acuerdo con Minerva (2006) se establece como criterio de desempeño el que "el estudiante clasifique o determine las variables de estudio en una población o muestra, y además, tabule y represente gráficamente un conjunto de datos asociados a su entorno y que elija la medida de tendencia central y variabilidad más adecuada".

En investigaciones realizadas por Dolores (2007, p. 69) a estudiantes de primaria y secundaria acerca de la lectura e interpretación de gráficas utilizadas por los medios de información y que van dirigidas a amplios sectores de la sociedad, se determinó que: "La mayoría de los alumnos identifican lo que cambia (las variables), hacen lectura de dato por dato o punto por punto (según corresponda), evidenciando un escaso conocimiento sobre los significados de los conceptos sociales representados en las gráficas socialmente compartidas". Esta problemática se hereda en el Nivel Medio Superior (NMS) y es totalmente evidente en la materia de Estadística, el mundo de vertiginosa información en que nos encontramos sumergidos demanda seres capaces de seleccionar aquella que le convenga, para mejorar sus condiciones de vida a partir de la toma de decisiones razonada; es decir seres estadísticamente cultos como lo menciona Arteaga, Batanero, Cañadas y Contreras (2011) dando lugar a la interrogante:

¿Cómo conseguir que la educación estadística básica sea una realidad para todos?

Como sugiere Gal (2002) citado por Arteaga, Batanero, Cañadas y Contreras (2011) las competencias que una persona debe desarrollar en pro de dicha educación son:

- Interpretar y evaluar críticamente la información estadística.

- Discutir o comunicar sus opiniones respecto a tales informaciones estadísticas cuando sea relevante.

Al constatar las graves deficiencias del estudiante de Estadística en la lectura de gráficas, surge la necesidad de reforzar en él, los procesos básicos de pensamiento mencionados por Novak (1988) como la observación, comparación, análisis y síntesis; necesarios en la interpretación de información que proyectan las gráficas de cualquiera que sea el trabajo de investigación.

Una vez clarificada la meta, ¿cómo alcanzarla? 
El modelo UbD (Understanding by Design) comprendiendo a través del diseño, permite centrar el diseño de la actividad escolar en el aprendizaje del alumno, es decir, es un modelo para la creación y el entendimiento. Sus autores Wiggins \& McTighe (1998) refieren que se trata de un marco conceptual, para la planificación del currículo, para el diseño de la evaluación y, la enseñanza para la comprensión y la transferencia. Se propicia la mejora en el desempeño estudiantil debido a que el profesor es el encargado de diseñar ambientes de trabajo colaborativo donde el estudiante:

- Logre un currículo efectivo, direccionado y orientado hacia el aprendizaje significativo.

- Desarrolle y profundice la comprensión.

- Aprenda a partir de grandes ideas.

- Logre las seis facetas de comprensión: explicar, interpretar, aplicar, cambiar de perspectiva, empatía y autoevaluación.

Como marcan Wiggins \& Tighe (2001) Understanding by Design es un modelo de "diseño inverso" o "trabajando hacia atrás" que consta de tres etapas y que debe responder los siguientes cuestionamientos:

- Etapa1 - Resultados deseados

¿Qué objetivos de transferencia a largo plazo se abordan? ¿Qué significados deben obtener los estudiantes con el fin de llegar a entendimientos importantes? ¿Qué preguntas esenciales van a explorar los estudiantes? ¿Qué conocimientos y habilidades van a adquirir los estudiantes? ¿Qué objetivos o estándares se cubren?

- Etapa 2 - Evidencia

¿Qué desempeños y productos mostrarán la evidencia del significado y de la transferencia? ¿Con qué criterios deberán evaluarse el desempeño, tomándose en cuenta los resultados deseados de la etapa 1? ¿Qué otro tipo de evidencias se recolectará para todos los resultados deseados de la Etapa 1? ¿Están las evaluaciones alineadas a todos los elementos de la Etapa 1?

- $\quad$ Etapa 3 - Plan de aprendizaje

¿Qué actividades, experiencias y lecciones permitirán el logro de los resultados deseados y el éxito en las evaluaciones? ¿Cómo va a ayudar el plan de aprendizaje a los estudiantes con la adquisición, el dar significado, y la transferencia? ¿Cómo se dará secuencia a la unidad y cómo se va a diferenciar para optimizar el rendimiento de todos los alumnos? ¿Cómo se va a monitorear el progreso? ¿Están alineadas las actividades de aprendizaje en la Etapa 3 con los objetivos de la Etapa 1 y las evaluaciones de la Etapa 2?

Con base a lo anterior en el presente trabajo se pretende que el estudiante a largo plazo sea capaz de redactar conclusiones que detallen el comportamiento de gráficas, empleando el lenguaje estadístico apropiado, los significados que generen entendimientos relevantes en el estudiante parten del hecho de que el alumno deduzca las preguntas óptimas que se deben realizar al analizar los resultados gráficos de una encuesta, para la generación de conclusiones que evidencien la lectura e interpretación de graficas contextualizadas, lo que conduce a recurrir a actividades generadoras porque a partir de un resultado, en este caso el análisis de la gráfica, se genera una pregunta.

Una actividad generadora es aquella que produce o da origen a una diversidad de respuestas, con semejanzas en el sentido matemático pero con determinadas variaciones en su estructura, que revelan información valiosa sobre las formas en que los estudiantes están pensando en la tarea o la comprensión que tiene sobre determinados objetos matemáticos.

La función de una actividad generadora resulta conveniente para el desarrollo del presente trabajo de investigación por la relevancia que conlleva conocer lo que sabe el estudiante y utilizarlo como base para promover nuevos aprendizajes, es decir contribuir al proceso de asimilación planteado por Ausubel (1983).

Ante el reto de formar ciudadanos estadísticamente cultos en la presente propuesta se comparte cómo el aplicar una actividad generadora a los estudiantes de nivel medio superior les ayuda a dar el gran primer paso, es decir el desarrollo de la competencia de lectura e interpretación crítica de tablas y gráficos a través de la construcción de un cuestionario. Misma que de acuerdo con Curcio (1989) provoca la aparición de tres niveles de lectura:

- Lectura entre datos: lectura literal del gráfico. 
- Lectura dentro de los datos: integra los datos que observa a través de la comparación.

- Leer más allá de los datos: realiza predicciones e inferencias con base a lo que observa.

METODOLOGÍA

¿En qué consiste la estrategia?

La técnica Elaboración de un Cuestionario es una actividad de aprendizaje que gira en torno a la observación de gráficas del dominio público, gráficas que atrapen la atención del estudiante, en este caso nuestro alumno al pertenecer al área de humanidades y ciencias sociales es cautivado con temas referentes a la lectura, parte de las fortalezas de este tipo de población estudiantil.

Del documento Primer informe sobre La encuesta Nacional de Lectura 2012 "De la penumbra a la oscuridad..." (FunLectura, 2012) se obtuvieron las gráficas que sirvieron como base para la aplicación de la estrategia. El estudiante debe deducir con base a lo observado las posibles preguntas que contestó la población encuestada, y lo más interesante es que el estudiante sea capaz de determinar las opciones de respuesta que debe incluir cada una de las preguntas elaboradas.

¿Cuándo hacer uso de la estrategia?

Debido a que se trata de una estrategia que se debe aplicar a la totalidad del grupo (72 estudiantes agrupados en equipos de 4 o 3 personas) y que debe redirigirse en momentos claves de la actividad, esta estrategia requiere aproximadamente 4 sesiones de 50 minutos.

¿Cómo hacer uso de la estrategia?

Antes de emplear la estrategia el estudiante debe tener claras las etapas que la integran:

$1^{a}$ Etapa. Resultados deseados. ( $1^{a}$ sesión)

Para la creación de un ambiente de aprendizaje adecuado es necesario que el profesor elija las gráficas cuyo contenido sea clave para la construcción del cuestionario, es decir aquellas que contengan información relevante tanto para el estudiante, como para el logro del objetivo. En nuestro caso las graficas pertinentes son:

\begin{tabular}{|c|c|c|}
\hline \multicolumn{3}{|c|}{$\begin{array}{l}\text { DE LA PENUMBRA A LA OSCURIDAD... } \\
\text { Encuesta Nacional de Lectura } 2012\end{array}$} \\
\hline CAPÍTULO & ETIQUETA & $\begin{array}{l}\text { TÍTULO DEL GRÁFICO } \\
\end{array}$ \\
\hline \multirow{4}{*}{$\begin{array}{l}\text { I. Datos } \\
\text { socioeconómicos }\end{array}$} & Gráfica A & Distribución de rangos de edad en años cumplidos. \\
\hline & Gráfica B & Ocupación actual de los entrevistados. \\
\hline & Gráfica C & Escolaridad (nivel máximo alcanzado). \\
\hline & Gráfica D & $\begin{array}{l}\text { Distribución por grupos de ingreso mensual en los } \\
\text { hogares. }\end{array}$ \\
\hline II. Capital Cultural & Gráfica 1 & $\begin{array}{l}\text { Último grado de estudios de los padres de los } \\
\text { entrevistados. }\end{array}$ \\
\hline \multirow{2}{*}{$\begin{array}{l}\text { III. Tipos de } \\
\text { materiales y } \\
\text { frecuencia de } \\
\text { lectura. }\end{array}$} & Gráfica 5 & $\begin{array}{l}\text { Libros leídos por los mexicanos mayores de } 12 \text { años } \\
\text { en los últimos } 6 \text { meses. }\end{array}$ \\
\hline & Gráfica 6 & Preferencias de lectura de los mexicanos. \\
\hline \multirow{2}{*}{$\begin{array}{l}\text { IV. Creación de } \\
\text { hábitos de lectura y } \\
\text { socialización }\end{array}$} & Gráfica 11 & Cantidad de libros en los hogares de los mexicanos. \\
\hline & Gráfica 12 & Relación entre cantidad de libros y lectura actual. \\
\hline \multirow{2}{*}{$\begin{array}{l}\text { V. Capacidades y } \\
\text { actitudes en relación } \\
\text { con la lectura }\end{array}$} & Gráfica 14b & ¿Ahora usted lee más o menos que antes? \\
\hline & Gráfica 15 & ¿Le gusta leer? \\
\hline $\begin{array}{l}\text { VI. Usos sociales de } \\
\text { la lectura }\end{array}$ & Gráfica 16a & $\begin{array}{l}\text { Relación entre tiempo dedicado a la lectura por gusto } \\
\text { y material de lectura preferido. }\end{array}$ \\
\hline $\begin{array}{l}\text { VII. Usos de las } \\
\text { tecnologías de la } \\
\text { información }\end{array}$ & Gráfica 22 & $\begin{array}{l}\text { Principales razones por las que los mexicanos utilizan } \\
\text { internet. }\end{array}$ \\
\hline
\end{tabular}




\begin{tabular}{|l|c|l|}
\hline $\begin{array}{l}\text { VIII. Asistencia a } \\
\text { espacios culturales }\end{array}$ & Gráfica 23 & Asistencia a bibliotecas públicas. \\
\hline $\begin{array}{l}\text { IX. Una mirada a la } \\
\text { evidencia... }\end{array}$ & $\begin{array}{l}\text { Última } \\
\text { gráfica }\end{array}$ & Bibliotecas de Aula y Escolares en Educación Básica. \\
\hline
\end{tabular}

Por ejemplo: Se pide a los estudiantes que:

- Elaboren el cuestionario referente a las gráficas del capítulo I (A, B y C)

- Que simulen aplicarlo a una población con el número de personas correspondientes y construyan una tabla con las respuestas manipulando los resultados de tal manera que al graficar en Excel resulte una gráfica similar a la original.
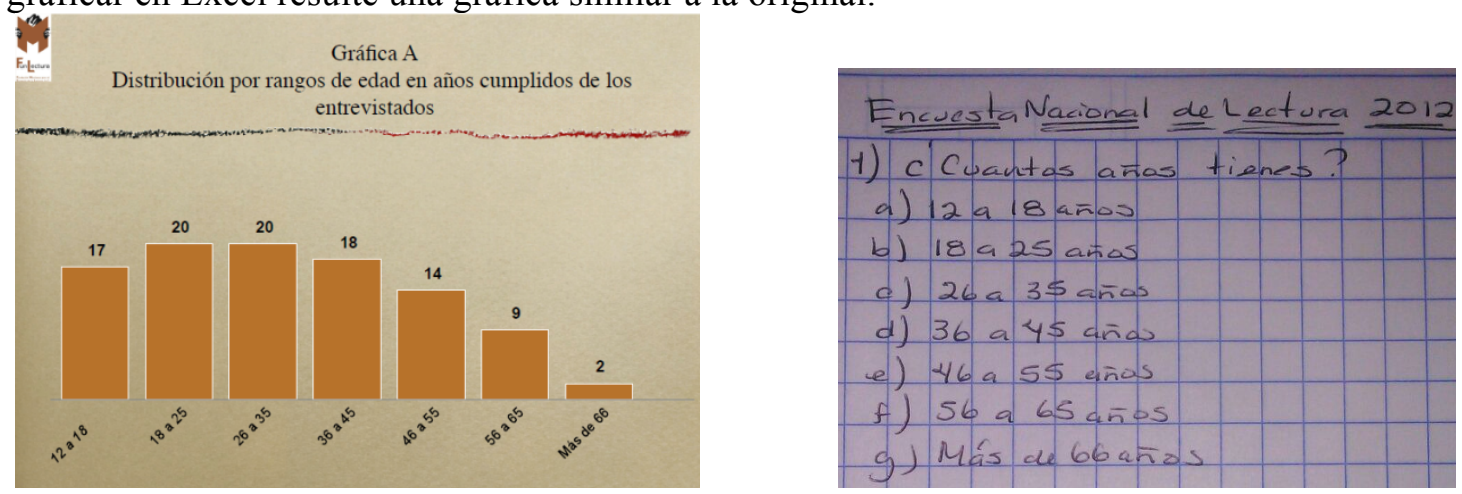

$2^{a}$ Etapa. Evidencia. (2 $2^{a}$ sesión)

1) Revisión de la tarea. A través de la tarea los estudiantes lograrán una autoevaluación porque serán capaces de verificar en qué medida sus resultados se aproximan a los originales. Y externar sus dudas en plenaria durante la $2^{\mathrm{a}}$ sesión. ( $10 \mathrm{~min}$.).

2) El desempeño que se espera en esta segunda etapa durante la sesión dos, es generar las preguntas y opciones de respuesta de todas las gráficas marcadas, se pide a los equipos concentrarse durante la sesión en las gráficas 14b, 15 y 16a con un tiempo límite de 20 min. Al paso de los 20 minutos en plenaria se analizan los resultados, con ayuda del maestro y a través de la técnica de la pregunta los equipos deben detectar que la diferencia entre las gráficas de tarea y las actualmente señaladas, radica en que intervienen más de una variable a analizar y como consecuencia cada gráfica generará más de una pregunta con su opción de respuesta.

3) La tarea recomendada a los equipos es simular en Excel la aplicación de las preguntas a las personas que convenga, generando una tabla de distribución de frecuencias y sus respectivas gráficas similares a la 14b, 15 y $16 \mathrm{a}$.

\section{$3^{a}$ Etapa. Plan de aprendizaje.}

$3^{a}$ sesión.

1) A través de la tarea los estudiantes lograrán una autoevaluación porque serán capaces de verificar en qué medida sus resultados se aproximan a los originales. Y externar sus dudas en plenaria durante la $3^{\mathrm{a}}$ sesión. (10 min.).

2) Se pide a los equipos que elaboren el cuestionario completo para todas las gráficas marcadas en la etapa 1. El producto debe estar terminado al final de la sesión y deberá ser entregado al profesor para su revisión. (30 min.).

$$
4^{a} \text { sesión. }
$$

1) Se entregan los cuestionarios revisados con las debidas correcciones en caso de así requerirlo. (10 min).

2) En plenaria se analizan las preguntas generadoras de las gráfica 16a, con la intervención del profesor y a través de lluvia de ideas, se propicia que los estudiantes establezcan las variables que determinan la gráfica, relacionándolas entre sí y conduciendo al grupo hacia la conclusión del comportamiento de la gráfica basada en las variables establecidas. Además los estudiantes reconocerán la existencia de conceptos matemáticos y cómo influye su comportamiento en el gráfico analizado. (15min). 
3) Cada equipo debe elaborar una conclusión que incluya las gráficas 14b, 15, 22 y 23, estableciendo las variables necesarias tomando como base las preguntas y respuestas del cuestionario obtenido. Además se pide agreguen a la conclusión un comentario final que refleje su punto de vista acerca de lo que ocurre en el Primer informe sobre La encuesta Nacional de Lectura 2012 "De la penumbra a la oscuridad..." (25 min.).

\section{ANÁLISIS DE RESULTADOS}

En el grupo se formaron 20 equipos en total, 12 equipos de 4 integrantes y 8 equipos de 3 estudiantes.

\section{Durante la $1^{a}$ sesión:}

- En el análisis de las Gráficas A, B, C, D después de los 20 minutos de reflexión el 40\% de los equipos construyeron las preguntas con las posibles opciones de respuesta en forma correcta, el 50\% sólo construyó de manera acertada las preguntas y omitieron la posibles respuestas a elegir y el $10 \%$ restante tuvo errores en la redacción de las preguntas, además de no considerar opciones para las respuestas.

\section{En la $2^{a}$ sesión:}

- En la revisión de la tarea, el 95\% de los equipos construyeron correctamente los cuestionarios con preguntas y opciones de respuesta, así como las tablas y graficas de Excel. El equipo restante tuvo problemas con el manejo de Excel.

- En el análisis de las gráficas $14 \mathrm{~b}, 15$ y $16 \mathrm{a}$, el $20 \%$ de los equipos detectaron todas las preguntas con algunos errores en las opciones de respuesta que generan las gráficas.

- El 30\% detectó más de una pregunta por gráfica, con algunos errores en las opciones de respuesta.

- El $50 \%$ de los equipos elaboraron sólo una pregunta por cada gráfica con errores en las opciones de respuesta.

Resultados de la $3^{a}$ sesión:

- En la revisión de la tarea, el 85\% de los equipos construyeron correctamente los cuestionarios con preguntas y opciones de respuesta, así como las tablas y gráficas de Excel, el 15\% de los equipos restantes tuvo problemas con el manejo de Excel al tratar de graficar más de una variable.

- El $80 \%$ de los equipos terminaron el cuestionario, el $20 \%$ restante lo envió por correo electrónico al profesor antes de terminar el día.

Resultados de la $4^{a}$ sesión:

En la revisión del cuestionario completo:

- El $35 \%$ de los equipos entregaron un cuestionario completo en número de preguntas y opciones de respuesta, sin errores.

- El $20 \%$ obtuvieron un cuestionario completo en número de preguntas y algunos errores en las opciones de respuesta.

- El 45\% entregaron incompleto el cuestionario, faltando cómo máximo 4 preguntas.

En la conclusión que incluye las gráficas $14 \mathrm{~b}, 15,22$ y 23 :

- El $60 \%$ de los equipos realizan una conclusión basada en la lectura e interpretación de las gráficas con el adecuado establecimiento de las variables, además de involucrar en su redacción cómo influyen conceptos matemáticos tales como promedio, moda, varianza y porcentajes en el comportamiento de los resultados.

- El $25 \%$ de los equipos redactaron conclusiones faltando alguna de las variables pero involucrando en su redacción conceptos matemáticos.

- El 15\% de los equipos redactaron conclusiones faltando alguna de las variables pero no involucraron en su redacción conceptos matemáticos.

Acerca del comentario final:

- El 70\% agregó un comentario aplicando la inferencia a los resultados obtenidos y el 30\% restante se abstuvo de comentar. 


\section{INTERPRETACIÓN Y CONCLUSIONES}

Basados en el modelo UbD "trabajando hacia atrás" y aplicando actividades generadoras "deducción del cuestionario" se logró incidir en el desempeño del estudiante de Estadística del área de Humanidades perteneciente a la Preparatoria Enrique Cabrera Barroso de la BUAP, al verificar que el $85 \%$ de la población construye conclusiones basadas en lecturas e interpretaciones de gráficas contextualizadas, clasificando las variables de estudio de la muestra poblacional, tabulando y representando gráficamente como parte de la autoevaluación promovida en las tareas de cada sesión y haciendo referencia a medidas de tendencia central y variabilidad, tal como lo propone el Plan 06 por Competencias. Al redactar la conclusión se observa que el estudiante logra aplicar la estadística descriptiva para inferir comportamientos de una población.

E1 15\% restante no cumple en su totalidad con los desempeños necesarios para la lectura e interpretación de gráficas, solo realiza lectura entre y dentro de los datos. Al final del proceso se observó que el estudiante muestra interés en la información grafica que le rodea, asimilándola y transmitiéndola de manera confiable a sus pares, además de observar en el estudiantado el logro de las capacidades para explicar, interpretar, aplicar, cambiar perspectiva, sentir empatía y autoevaluarse; indicadores del entendimiento.

Los resultados obtenidos en el trabajo desarrollado con los estudiantes de Humanidades de la PECU de la BUAP muestran como la implementación de actividades generadoras en el modelo UbD provocan en el estudiante la potenciación de las habilidades básicas de pensamiento; para que, a partir de la observación pueda comparar, analizar y sintetizar información estadística necesaria y así, redactar conclusiones que revelen una adecuada lectura, interpretación de gráficas y predicción de resultados, en otras palabras la lectura más allá de los datos.

\section{REFERENCIAS}

Arteaga, P., Batanero, C., Cañadas, G. y Contreras M. (2011). Las Tablas y Gráficos Estadísticos como Objetos Culturales. Números. Revista de Didáctica de las Matemáticas , 56-67.

Ausubel, N. H. (1983). Psicología Educativa: Un punto de vista cognoscitivo. México: $2^{\circ}$ Ed. Trillas.

Carmona, G., Dominguez, A., Krause, G., \& Durán, P. (2011). Emergent public spaces: Generative activities on function interpolation. Contemporary Issues in Technology and Teacher Education, 11(4), 362-381.

Curcio, F. R. (1989). Developing graph comprehension. Reston, VA: N.C.T.M

Dolores, C. (2007). Lectura e interpretación de gráficas socialmente compartidas. Relime, 10(1), 69-96.

FunLectura. (2012). Encuesta Nacional de Lectura 2012. De la penubra a la oscuridad. México: Fundación Mexicana para el Fomento de la Lectura, A.C.

Godino, J. D. (2002). Un enfoque ontológico y semiótico de la cognición matemática. Recherches en Didactique des Mathématiques, 22(2/3), 237-284.

Minerva, M. U. (2006). Plan de Estudios 06 Nivel Medio Superior. Recuperado el 14 de Noviembre de 2014, de Benemérita Universidad Autónoma de Puebla: http://www.buap.mx/portal_pprd/work/sites/Educacion_media/resources/PDFContent/43/plan6 docuemnto.pdf

Novak, Joseph y Gowin, Bob. (1988). Aprendiendo a aprender. Barcelona, España: Martínez Roca.

Wiggins, G. \& Mc Tighe, J. (1998). Understanding by design. Alexandria, VA: Association for Supervision and Curriculum Development

Wiggins, G., \& McTighe, J. (2011). The Understanding by Design Guide to Creating High-Quality Units. Alexandria, Virginia USA: ASCD.

Wray, David y Lewis, Maureen. (2000). Aprender a leer y escribir textos de información. Madrid, España: Morata. 